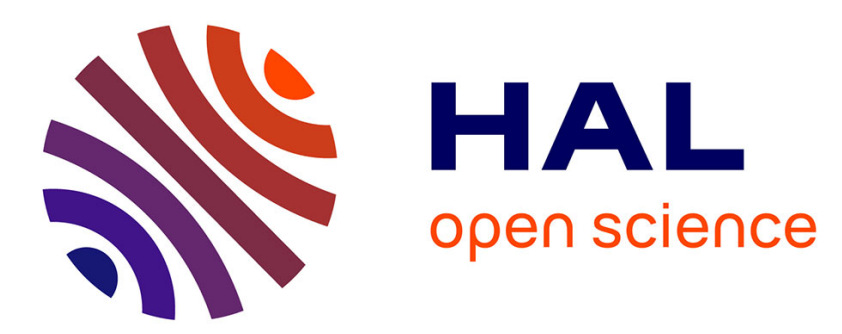

\title{
How to Set the Fractional Power Control Compensation Factor in LTE?
}

Marceau Coupechoux, Jean-Marc Kélif

\section{To cite this version:}

Marceau Coupechoux, Jean-Marc Kélif. How to Set the Fractional Power Control Compensation Factor in LTE?. IEEE Sarnoff Symposium, May 2011, Princeton, United States. pp.1-5. hal00721232

\section{HAL Id: hal-00721232 https://hal-imt.archives-ouvertes.fr/hal-00721232}

Submitted on 27 Jul 2012

HAL is a multi-disciplinary open access archive for the deposit and dissemination of scientific research documents, whether they are published or not. The documents may come from teaching and research institutions in France or abroad, or from public or private research centers.
L'archive ouverte pluridisciplinaire HAL, est destinée au dépôt et à la diffusion de documents scientifiques de niveau recherche, publiés ou non, émanant des établissements d'enseignement et de recherche français ou étrangers, des laboratoires publics ou privés. 


\title{
How to Set the Fractional Power Control Compensation Factor in LTE ?
}

\author{
Marceau Coupechoux ${ }^{1}$, Jean-Marc Kelif ${ }^{2}$ \\ ${ }^{1}$ TELECOM ParisTech \& CNRS LTCI, Paris, France \\ coupecho@enst.fr \\ ${ }^{2}$ Orange Labs, Issy-Les-Moulineaux, France \\ jeanmarc.kelif@ orange-ftgroup.com
}

\begin{abstract}
The uplink power control procedure in Long Term Evolution (LTE) cellular networks is made of an open-loop part and a closed loop part. In this paper, we focus on the former and study the compensation factor of the related Fractional Power Control (FPC) scheme. In particular, we propose a first analytical approach in order to derive approximate equations for the Signal to Interference plus Noise Ratio (SINR) at a given distance of the eNode-B, the average SINR, and the average cell spectral efficiency. This method avoids extensive and timeconsuming simulations. From derived expressions, we are able to find the optimal compensation factor, and to study the impact of various network and environment parameters on the system performance.
\end{abstract}

\section{INTRODUCTION}

Power control plays an important role on the uplink of Long Term Evolution (LTE) cellular networks. The standard [1] has defined a combination of open loop and closed loop power control. The open loop part of the scheme is often called Fractional Power Control (FPC) because it allows User Equipments (UE) to partially compensate the path-loss. FPC is characterized by two main parameters: a target received power $P_{0}$ and a compensation factor $\alpha$. In this paper, we focus on the open loop algorithm and on the compensation factor and we propose an analytical approach for studying this parameter.

In literature, different papers have studied the power control of LTE in various environments and with various parameters settings.

In [2], authors study by simulations the influence of the fractional power control parameters $P_{0}$ and $\alpha$ (described in details in the following section). They show the increase of the Signal to Interference plus Noise Ratio (SINR) standard deviation with $\alpha$. They also study in depth the influence of $P_{0}$ for a fixed value of $\alpha=0.6$ and show how this power can be optimized in order to get higher cell-edge throughput and average cell throughput.

Authors of [3], [4] compare the pure open loop power control scheme with a combination of open and closed loop algorithms. They study the influence of the maximum terminal power and of the dynamic Radio Blocks (RB) allocation. Performances are obtained through extensive simulations for some combinations of $P_{0}$ and $\alpha$ in [3] and a joint optimization of these two parameters is performed in [4].

Analysis performed in [5], [6] show that closed loop power control combined with FPC can greatly improve performances achieved by a pure open loop scheme. Based on simulations, authors claim that $\alpha=0.8$ is an optimal value. They however study only three possible values for $\alpha: 0.7,0.8$ and 1 .

In [7], authors perform system level simulations in order to propose sub-optimal configurations for the fractional power control algorithm. The key point of their study is to maintain a constant interference power by increasing $P_{0}$ when $\alpha$ is decreased. With their proposed setting, they point out the gain of FPC over a full compensation power control.

Authors of [8] compare also by simulations FPC with closed loop power control but only for a limited number of $\alpha$ values and for specific target SINR.

Other older references ([9], [10]) are also based on simulations.

In this paper, contrary to the rest of the literature, we propose an analytical approach for the study of FPC compensation factor, which allows to establish instantaneous results. To the best of our knowledge, all previous papers on the subject rely on extensive and time-consuming simulations. The paper is organized as follows. Section II presents the FPC in LTE; section III presents our network model and derive equations for the SINR and the cell spectral efficiency; in section IV, numerical results are provided. At last, the last section concludes the paper.

\section{Fractional Power Control in LTE}

Power control has been a major radio feature of WCDMA cellular networks. In such systems, in particular on the uplink, fast power control follows the variations of the channel (pathloss, shadowing and fast fading) in order to limit as much as possible intra-cell interference. With HSPA, fast power control is abandoned on the downlink (HSDPA) and channel variations are rather taken into account by the fast link adaptation and scheduling. This paradigm change is facilitated by the multiple access scheme, which is a mixed of CDMA (code domain) and TDMA (time division in Time Transmit Intervals - TTI). Fast power control is however still present on the uplink (HSUPA).

Contrary to previous 3GPP standards, LTE completely abandons fast power control both on the downlink and on the uplink. The resources allocated to different users of a given cell are indeed orthogonal. With OFDMA on the downlink or with SC-FDMA on the uplink, there is no (or limited if we consider inter-carrier interference) intra-cell interference. As 
a consequence, power control in LTE is slow (it follows the variations of the path-loss and of the shadowing) and has the main goal of limiting or controlling inter-cell interference, in particular for cell edge users. As in HSDPA, fast variations of the radio channels are considered by the fast link adaptation scheme and the scheduler.

On the uplink, power control is responsible for setting the UE transmitted power per SC-FDMA symbol. Its main goal is to reduce the power emitted by UEs that are important sources of interference for neighbor cells, i.e., UEs at the cell edge. It consists in two parts: an open loop scheme based on UE own measurements; and a closed loop scheme based on commands sent by the eNode-B. On the uplink data shared channel PUSCH, UE transmitted power $P_{P U S C H}$ can be written in $\mathrm{dBm}[1]$ :

$P_{P U S C H}=\min \left\{P_{\max }, 10 \log (M)+P_{0}+\alpha P L+\Delta_{T F}+f\right\}$

where:

- $P_{\max }$ is the maximum transmit power of the UE;

- $M$ is the number of Radio Blocks (RB) allocated to the UE;

- $P_{0}$ is a target received power at eNode-B;

- $\alpha$ is called the compensation factor;

- $P L$ is the UE-eNode-B path-loss;

- $\Delta_{T F}$ is a corrective factor depending on the Transport Format;

- $f$ is a closed loop command issued by the eNode-B.

In this paper, we analyze the influence of the compensation factor $\alpha$, we thus ignore $P_{\max }, \Delta_{T F}$ and $f$, we consider a single RB $(M=1)$ and we impose a common figure for $P_{0}$ for all UE of a cell and for all cells. In this simplified case, transmit power $P_{T x}$ can be written (in $\mathrm{dBm}$ ):

$$
P_{T x}=P_{0}+\alpha P L
$$

and the received power $P_{R x}$ at the eNode-B:

$$
P_{R x}=P_{0}+\alpha P L-P L=P_{0}+(\alpha-1) P L .
$$

At this stage, we can distinguish several cases:

- $\alpha=1$ : we have here a classical power control scheme similar to the one implemented in CDMA (except that the power control is slow). The scheme totally compensates the path-loss in order to reach the target received power $P_{0}$. All UE of the cell, whatever their location, see their signals received with the same power at eNode-B. We are in presence of a full compensation power control.

- $\alpha=0$ : transmission power is here fixed and does not depend on the path-loss. There is no compensation and in fact no power control at all. UE closed to the eNode$\mathrm{B}$ are received with high power while cell edge UE are received with a weak signal.

- $0<\alpha<1$ : we are in the case of a fractional power control, where path-loss is partially compensated by the power control scheme. The higher is the path-loss, the smaller is the received power at eNode-B. Cell edge UEs are thus received with a weaker signal. They however create less inter-cell interference because their transmit power is also reduced.

We thus see that increasing $\alpha$ increases the received power of cell edge UE but also the inter-cell interference. How to set $\alpha$ in order to maximize the cell capacity is the question we would like to answer in the next sections.

\section{SINR AND SPECTRAL EFFICIENCY}

In this section, we present our analytical approach for the study of the FPC compensation factor.

\section{A. Network and Propagation Model}

In this paper, we consider a homogeneous cellular LTE network made of $B$ eNodes-B, and we focus on the uplink. eNodes-B have omni-directional antennas, so that one eNodeB covers a single cell. Half-distance between eNodes-B is denoted $R_{c}$. Frequency reuse 1 is assumed, all eNode-B transmit on the whole system bandwidth ${ }^{1}$. UEs are supposed to be uniformly distributed on the cell areas. They are assumed to have traffic of packet type, so that their throughput is an increasing function of their Signal to Interference plus Noise Ratio (SINR).

Let us consider a particular UE $u$ located in cell $b=0$. According to the FPC scheme, the received power of $u$ at eNode-B can be written (in $\mathrm{mW}$ ):

$$
S_{u}=p_{0} g_{u, 0}^{1-\alpha},
$$

where $P_{0}=10 \log \left(p_{0}\right)$ ( $P_{0}$ is the target received power in $\mathrm{dBm}$ and $p_{0}$ in $\mathrm{mW}$ ) and $g_{u, b}$ is the path-gain between UE $u$ and eNode-B $b$.

On one $\mathrm{RB}$, user $u$ suffers from the interference of a single UE in each of the network cells. In absence of inter-cell coordination, interfering UE can be located anywhere in the interfering cells. External interference can thus be written:

$$
I_{e x t}=\sum_{b=1}^{B-1} p_{0} g_{v(b), b}^{-\alpha} g_{v(b), 0},
$$

where $v(b)$ designates the UE scheduled by eNode-B $b$. The SINR of user $u$ is then $\gamma_{u}=S_{u} /\left(I_{e x t}+N\right)$, where $N$ is the thermal noise power in the system bandwidth.

While considering a urban environment, known to be interference limited, the SINR can be approximated by $\gamma_{u} \approx$ $S_{u} / I_{\text {ext }}$.

In this paper, we assume that the path-gain $g_{u, b}$ is only depending on the distance between $u$ and $b$ and can be written $g_{u, b}(r)=K r^{-\eta}$, where $K$ is a constant, $r$ is the distance between $u$ and $b$ and $\eta$ is the path-loss parameter ${ }^{2}$.

\footnotetext{
${ }^{1}$ This assumption can be easily relaxed by slightly adapting our model, as it has been shown on the downlink in [11]

${ }^{2}$ The introduction of shadowing is left for further work. We have shown in [12] that it can be integrated in our model for the downlink.
} 


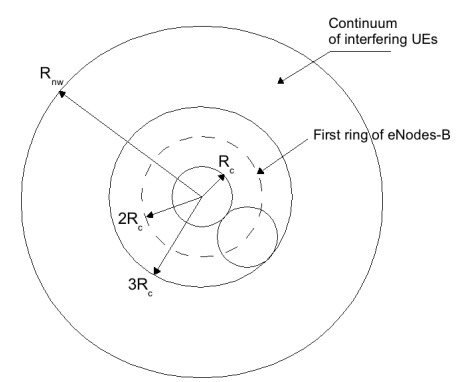

Figure 1. Illustration of the considered network model for interference calculation.

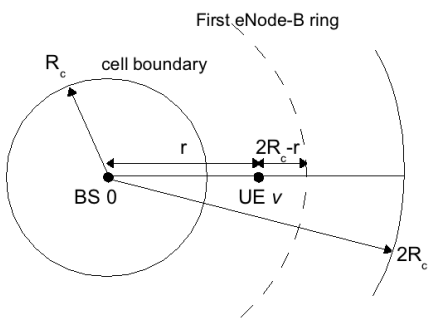

Figure 2. Illustration of the distances involved in the computation of the interference due to a UE $v$ located at distance $r \in\left[R_{c} ; 2 R_{c}\right]$ from the central eNode-B.

\section{B. Interference Derivation}

Whereas the expression 1 can be easily obtained with the considered propagation model, the expression of $I_{e x t}$ is not easy to handle since it depends on the location of all interfering UEs. We thus propose in this subsection an easy-to-handle formula that will allow us to study the $\alpha$ parameter.

The key modeling step of the model we propose consists in replacing a given fixed finite number of interfering transmitters by an equivalent density of transmitters distributed according to some distribution function. Our model, called the fluid model in [12], is illustrated in Fig. 1: the central cell is modeled by a disk of radius $R_{c}$ and is surrounded by several rings of interfering cells at distances $2 n R_{c}(n=1,2, \ldots)$. The network size can be expressed as $R_{n w}=\left(2 N_{c}+1\right) R_{c}$, where $N_{c}$ represents the number of rings.

Note that the first ring of cells is located between distances $R_{c}$ and $3 R_{c}$, the second ring between $3 R_{c}$ and $5 R_{c}$ and the $n^{t h}$ ring between $(2 n-1) R_{c}$ and $(2 n+1) R_{c}$.

Considering a uniform UE density $\rho_{U E}$, expression 2 can be approximated as:

$$
I_{e x t}=\int_{R_{c}}^{R_{n w}} \int_{0}^{2 \pi} \rho_{U E} p_{0} g_{v(b), b}^{-\alpha} g_{v(b), 0} r d r d \theta .
$$

In order to integrate this expression, let us focus on a UE $v$ located in the first ring of interfering cells at distance $r \in$ $\left[R_{c} ; 2 R_{c}\right]$ from eNode-B 0 , as illustrated in Fig. 2. In first order, UE $v$ is located at distance $2 R_{c}-r$ from its eNode-B. Thus, the interference created by the small area $2 \pi r d r$ around UE $v$ is $\rho_{U E} p_{0} K^{-\alpha}\left(2 R_{c}-r\right)^{\alpha \eta} K r^{-\eta} 2 \pi r d r$.

In the same way, if UE $v$ is located at distance $r \in$ $\left[2 R_{c} ; 3 R_{c}\right]$, it is, in first order, at distance $r-2 R_{c}$ from its
eNode-B. Thus, the interference created by the small area $2 \pi r d r$ around UE $v$ is $\rho_{U E} p_{0} K^{-\alpha}\left(-2 R_{c}+r\right)^{\alpha \eta} K r^{-\eta} 2 \pi r d r$.

This reasoning can be applied to any interfering ring, so that we can deduce the contribution of the $n^{t h}$ ring to the external interference:

$$
\begin{gathered}
I_{n, e x t}=2 \pi \int_{(2 n-1) R_{c}}^{2 n R_{c}} \rho_{U E} p_{0} K^{-\alpha}\left(2 n R_{c}-r\right)^{\alpha \eta} K r^{-\eta} r d r \\
+2 \pi \int_{2 n R_{c}}^{(2 n+1) R_{c}} \rho_{U E} p_{0} K^{-\alpha}\left(-2 n R_{c}+r\right)^{\alpha \eta} K r^{-\eta} r d r .
\end{gathered}
$$

For the first integral, we denote $x=1-\frac{r}{2 n R_{c}}$ and for the second one we denote $x=-1+\frac{r}{2 n R_{c}}$ so we obtain:

$$
\begin{aligned}
I_{n, e x t}= & 2 \pi \rho_{U E} p_{0} K^{1-\alpha}\left(2 n R_{c}\right)^{\alpha \eta+2-\eta} \times \\
& \int_{0}^{\frac{1}{2 n}} x^{\alpha \eta}\left[(1-x)^{1-\eta}+(1+x)^{1-\eta} d x\right]
\end{aligned}
$$

Finally, denoting

$$
I_{n}(\alpha, \eta)=\int_{0}^{\frac{1}{2 n}} x^{\alpha \eta}\left[(1-x)^{1-\eta}+(1+x)^{1-\eta}\right] d x,
$$

we can express the contribution of the $n^{\text {th }}$ ring as:

$$
I_{n, \text { ext }}=2 \pi \rho_{U E} p_{0} K^{1-\alpha}\left(2 n R_{c}\right)^{\alpha \eta+2-\eta} I_{n}(\alpha, \eta) .
$$

The total interference power is thus given by:

$$
I_{\text {ext }}=\sum_{n=1}^{N_{c}} I_{n, e x t} .
$$

\section{SINR and Spectral Efficiency Formulations}

From the previous study, we can deduce the SIR of user $u$ located at distance $r$ from eNode-B 0. From (1), we can express:

$$
S_{u}=p_{0} K^{1-\alpha} r^{-\eta(1-\alpha)} .
$$

1) SINR expression: Using (6) and (7), the SINR can be expressed, for an infinite network, as:

$$
\gamma_{u}(r)=\frac{r^{-\eta(1-\alpha)}}{2 \pi \rho_{U E} \sum_{n=1}^{\infty}\left(2 n R_{c}\right)^{\alpha \eta+2-\eta} I_{n}(\alpha, \eta)} .
$$

Remark 1: Denoting $\nu=\frac{r}{2 R_{c}}$ and since $\rho_{U E}=\frac{1}{\pi R_{c}^{2}}$ this expression can be written:

$$
\gamma_{u}(\nu)=\frac{\nu^{-\eta(1-\alpha)}}{8 \sum_{n=1}^{\infty} n^{\alpha \eta+2-\eta} I_{n}(\alpha, \eta)} .
$$

Expression (10) allows to establish that the uplink SINR does not explicitly depend on the location $r$ of the UE in the cell. It only depends on the relative location $\nu$ of the UE in the cell. Moreover this expression does not explicitly take into account the size $R_{c}$ of a cell.

Remark 2: We can obtain a simpler formula for $\alpha=0$ (no power control) because $I_{n}(\alpha, \eta)$ in (9) can be integrated:

$\gamma_{u}(r)=\frac{r^{-\eta}(\eta-2)}{2 \pi \rho_{U E}\left(2 R_{c}\right)^{2-\eta} \sum_{n=1}^{\infty}\left[\left(n-\frac{1}{2}\right)^{2-\eta}+\left(n+\frac{1}{2}\right)^{2-\eta}\right]}$. 
2) Average SINR: The average SINR over the cell area can be obtained by integration of (9) over a disk of radius $R_{c}$ :

$$
\begin{aligned}
\bar{\gamma} & =\int_{r_{0}}^{R_{c}} \gamma_{u}(r) \frac{2 \pi r d r}{\pi\left(R_{c}^{2}-r_{0}^{2}\right)} \\
& =\frac{\frac{R_{c}^{2-\eta(1-\alpha)}-r_{0}^{2-\eta(1-\alpha)}}{\left(R_{c}^{2}-r_{0}^{2}\right)(2-\eta(1-\alpha))}}{2 \pi \rho_{U E} \sum_{n=1}^{\infty}\left(2 n R_{c}\right)^{\alpha \eta+2-\eta} I_{n}(\alpha, \eta)} \\
& =\frac{1-\left(r_{0} / R_{c}\right)^{2-\eta(1-\alpha)}}{\left(1-\frac{r_{0}^{2}}{R_{c}^{2}}\right)(2-\eta(1-\alpha)) 2 \pi \sum_{n=1}^{\infty}(2 n)^{\alpha \eta-\eta} I_{n}(\alpha, \eta)}
\end{aligned}
$$

where $r_{0} \neq 0$ represents the minimum distance between a UE and its serving eNode-B. We notice that this expression assumes $\alpha \neq \frac{\eta-2}{\eta}$. For $\alpha=\frac{\eta-2}{\eta}$, we have:

$$
\bar{\gamma}=\frac{\ln \left(\frac{R_{c}}{r_{0}}\right)}{2 \pi\left(1-\frac{r_{0}^{2}}{R_{c}^{2}}\right) \sum_{n=1}^{\infty} I_{n}(\alpha, \eta)}
$$

3) Spectral efficiency: At last, the cell (or average) spectral efficiency (in bps/Hz/cell) is obtained using the classical Shannon formula:

$$
\bar{C}=\frac{2}{R_{c}^{2}-r_{0}^{2}} \int_{r_{0}}^{R_{c}} \log _{2}\left(1+\gamma_{u}(r)\right) r d r .
$$

We also define the spectral efficiency at distance $r: C(r)=$ $\log _{2}\left(1+\gamma_{u}(r)\right)$.

\section{Performance Evaluation}

In this section, we study the influence of different system and environment parameters on the network performance.

\section{A. Optimal $\alpha$}

We first study the influence of $\alpha$ on the cell efficiency. We set $r_{0}=50 \mathrm{~m}, R_{c}=1 \mathrm{Km}, \eta=3.5$ and we plot the cell spectral efficiency $\bar{C}$ as a function of $\alpha$ in Fig. 3. We also represent the spectral efficiency close to the eNode- $\mathrm{B}, C\left(r_{0}\right)$, and at cell-edge, $C\left(R_{c}\right)$.

We can first observe on the left of the figure that there is a clear trade-off between cell-edge users on the one hand and UEs close to the eNode-B on the other hand. As $\alpha$ is increasing, the spectral efficiency for close UEs decreases whereas it increases for cell-edge users. It is thus a very good means to improve the throughput of cell-edge users or to avoid outage at cell edge. Going from $\alpha=0$ to $\alpha=1$ indeed induces a $121 \%$ gain in terms of spectral efficiency for cell-edge users.

The decrease of performance for close UEs and the related increase for cell-edge UEs implies the existence of an optimal fractional power control parameter $\left(\alpha^{*}\right)$ for the cell spectral efficiency on the uplink, as illustrated on the right of the figure. With the chosen parameters, $\alpha^{*}=0.1$. At this optimal point, there is $46 \%$ gain in efficiency with respect to the full compensation case $(\alpha=1)$ and a $2.7 \%$ gain over the case without power control $(\alpha=0)$. The cell spectral efficiency reaches $\bar{C}=2.63 \mathrm{bps} / \mathrm{Hz} /$ cell in the best situation.

It is thus up to the cellular operator to set $\alpha$ either to $\alpha^{*}$ in order to get the best of its network or to any value of $\alpha>\alpha^{*}$
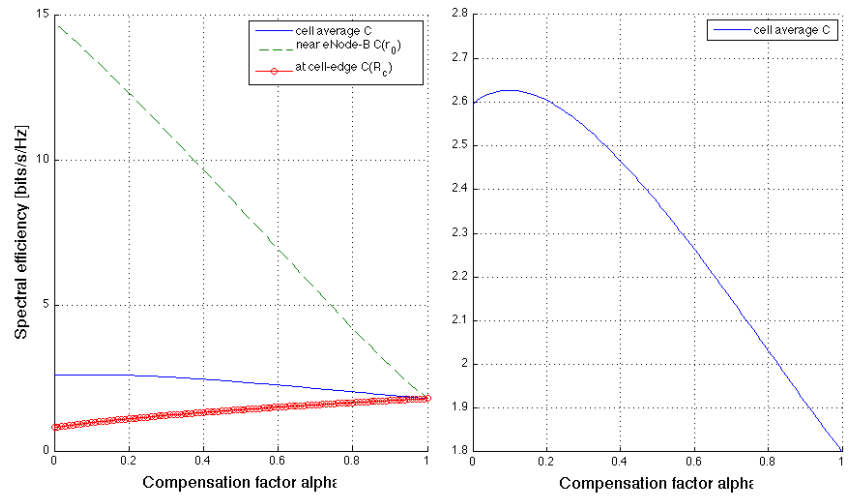

Figure 3. Spectral efficiency near the eNode-B (at distance $r_{0}$ ), at cell edge (at distance $R_{c}$ ) and in average over the cell area as a function of the compensation factor $\alpha$ (left); zoom for the cell spectral efficiency (right).
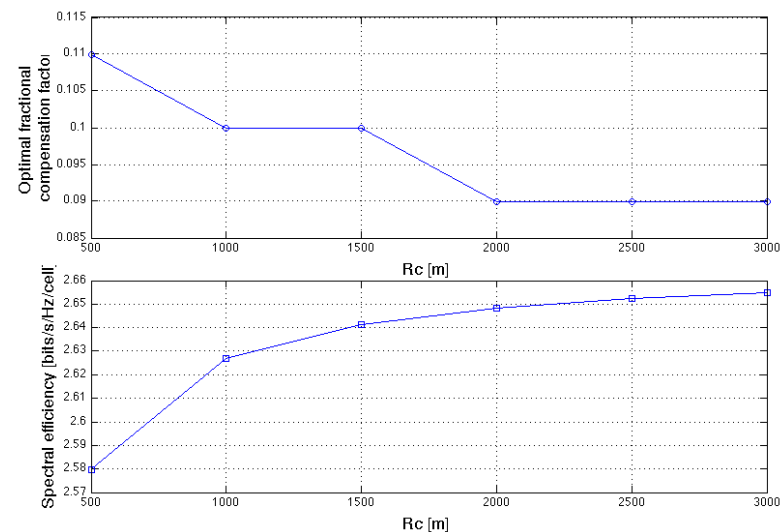

Figure 4. Influence of the half-distance between eNodes-B $R_{c}$ on the optimal compensation factor $\alpha^{*}$ (top); on the optimal cell spectral efficiency (bottom).

in order to increase the cell-edge UEs situation at the price of a reduced cell spectral efficiency.

Let us now analyze the influence of two important parameters: the half-distance between eNodes-B $R_{c}$ and the path-loss coefficient $\eta$.

\section{B. Cell Range Influence}

We have seen that there is no influence of $R_{c}$ on the SINR $\gamma_{u}$, according to (10). Only the relative distance from UE to eNode-B has an influence on the SINR. Parameter $R_{c}$ plays however a role in the expression of the average SINR (equation 12) and in the cell spectral efficiency (equation 14).

We see however in Fig. 4 (with $r_{0}=50 \mathrm{~m}$ and $\eta=3.5$ ) that the influence on the spectral efficiency and on the optimal $\alpha$ is tiny. Spectral efficiency is slightly increasing with $R_{c}, \alpha^{*}$ is slightly decreasing with $R_{c}$.

Note that in this paper we have neglected thermal noise in the system bandwidth (we assume a urban environment dominated by interference), this assumption can thus explain the small influence of $R_{c}$. Conclusions will probably change if noise is taken into account. 


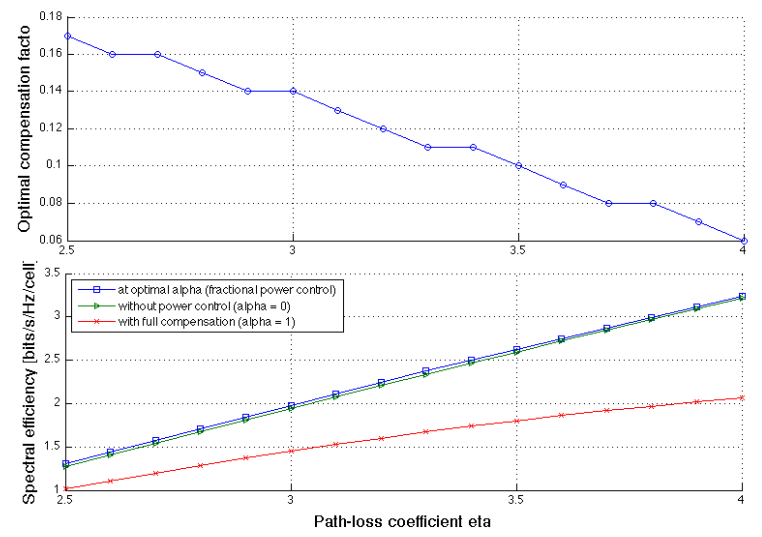

Figure 5. Influence of the path-loss coefficient $\eta$ on the optimal compensation factor $\alpha^{*}$ (top); and on the cell spectral efficiency (bottom).

\section{Path Loss Coefficient Influence}

The path-loss coefficient $\eta$ has a much higher influence on the system performance and on the optimal compensation factor $\alpha^{*}$. Cell spectral efficiency $\bar{C}$ and optimal $\alpha$ are shown in Fig. 5 as functions of $\eta\left(r_{0}=50 \mathrm{~m}\right.$ and $\left.R_{c}=1 \mathrm{Km}\right)$.

It is well known that cell capacity increases with $\eta$. The three curves (at optimum, with and without power control) are increasing functions of $\eta$. What is however striking is the fact that the gain obtained by tuning $\alpha$ to the optimum is relatively small compared to the no power control case whatever the path-loss coefficient. The gain over the full compensation case is however huge and is increasing with $\eta(+75 \%$ at $\eta=4)$.

\section{CONCLuSion}

In this paper, we propose for the first time an analytical approach for the study of the compensation factor of FPC in LTE networks. We derive approximate Signal to Interference plus Noise Ratio (SINR) and cell spectral efficiency equations that allow us to find the optimal factor for different network and environment configurations.

We show in particular that the optimal compensation factor slightly depends on the half-distance between eNodes-B but it is highly dependent on the path-loss coefficient. In terms of cell spectral efficiency, we can observe huge gains of Fractional Power Control (FPC) over full compensation power control. The gain is however tiny over the no power control case. Like previous works on the subject, we have highlighted and quantified the trade-off between cell-edge spectral efficiency and cell spectral efficiency.

The illustration of our analytical approach has been performed in a urban interference limited environment. We intend to extend our work by studying the impact of noise, of shadowing and of other FPC parameters like the maximum transmit power or the target received power.

\section{REFERENCES}

[1] 3GPP. 36.213 Physical Layer Procedures.

[2] N. J. Quintero. Advanced Power Control for UTRAN LTE Uplink. Master's thesis, Aalborg University, 2008.
[3] R. Müllner and C. F. Ball and K. Ivanov and J. Lienhart and P. Hric. Contrasting Open-Loop and Closed-Loop Power Control Performance in UTRAN LTE Uplink by UE Trace Analysis. In Proc. of IEEE Communications Conference ICC, 2009.

[4] R. Müllner and C. F. Ball and K. Ivanov and J. Lienhart and P. Hric. Performance Comparison between Open-Loop and Closed-Loop Uplink Power COntrol in UTRAN LTE Networks. In Proc. of ACM Int. Conf. on Wireless Communications and Mobile COmputing, IWCMC, 2009.

[5] B. Muhammad and A. Mohammed. Performance Evaluation of Uplink Closed Loop Power Control for LTE System. In Proc. of IEEE Vehicular Technology Conference VTC Fall, 2010.

[6] B. Muhammad. Closed Loop Power Control for LTE Uplink. Master's thesis, Blekingz Institute of Technology, 2008. Master of Science thesis.

[7] C. U. Castellanos and D. L. Villa and C. Rosa and K. I. Pedersen and F. D. Calabresse and P. Michaelsen and J. Michel. Performance of Uplink Fractional Power Control in UTRAN LTE. In Proc. of IEEE Vehicular Technology Conference VTC Spring, 2008.

[8] A. Simonsson and A. Furuskär. Uplink Power Control in LTE Overview and Performance. In Proc. of IEEE Vehicular Technology Conference VTC Fall, 2008.

[9] W. Xiao and R. Ratasuk and A. Ghosh and R. Love and Yakun Sun and R. Nory. Uplink Power Control, Interference Coordination and Resource Allocation for 3GPP E-UTRA. In Proc. of IEEE Vehicular Technology Conference, VTC Fall, 2006.

[10] A. M. Rao. Reverse Link Power Control for Managing Inter-Cell Interference in Orthogonal Multiple Access Systems. In Proc. of IEEE Vehicular Technology Conference, VTC Fall, 2007.

[11] M. Maqbool, Ph. Godlewski, M. Coupechoux, and J.-M. Kélif. Analytical performance evaluation of various frequency reuse and scheduling schemes in cellular ofdma networks. Performance Evaluation, August 2009.

[12] J. M. Kélif and M. Coupechoux. Impact of topology and shadowing on the outage probability of cellular networks. In IEEE International Conference on Communications, ICC, Dresden, Germany, June 2009. 